\title{
Performance Evaluation of Routing Protocols for MAC Layer Models
}

\author{
Somya Jain ${ }^{1}$, Deepak Aggarwal ${ }^{2}$ \\ ${ }^{1}$ (Division of Computer Engineering, Netaji Subas Institute of Technology University of Delhi, India) \\ ${ }^{2}$ (Division of Computer Engineering, Maharishi Dayanand University, India)
}

\begin{abstract}
Mobile Ad-hoc Network (MANET) special characteristics is an inestimable addition to traditional networking technologies, and is presently under active research \& development.MANET are basically peer-topeer, multihop network which is a group of wireless mobile hosts forming a transient network that is having no fixed infrastructure. This paper encompasses the key studies carried out so far and describes all the parameters for simulation. In particular, draw comparisons among different routing protocols: Bellman-Ford, AODV, DSR, LAR1, WRP, FSR and ZRP based on the performance metrics like PDR, end-to-end delay and throughput. The study is carried out to investigate various statistics and highlights behavior of different routing protocols for several MAC layer protocols such as 802.11, CSMA, TSMA which gives rise to various performance issues using GloMoSim simulation environment. Finally, results show the unusual behavior when varying the number of nodes in the network layout and effects of switching among datalink models.
\end{abstract}

Keywords - ROUTING PROTOCOLS, AODV, DSR, LARI, WRP, ZRP

\section{INTRODUCTION}

MANET is a type of ad-hoc network with rapidly changing topology. These networks connect hundreds to thousands of nodes which are highly mobile. Practically, all the nodes behave as routers and take part in discovery and maintenance of routes to other nodes in the network. They are suited for use in situations where an infrastructure is unavailable or to deploy one is not cost effective. They have been applied to number of areas including virtual navigation, military, battlefield, personal area network and in different scenarios of education and entertainment. Routing protocol is that specifies how routers communicate with each other to disseminate information that allows them to select routes between any two nodes on a network. Ad-hoc Routing protocols can be divided broadly into three categories (Figure 1).

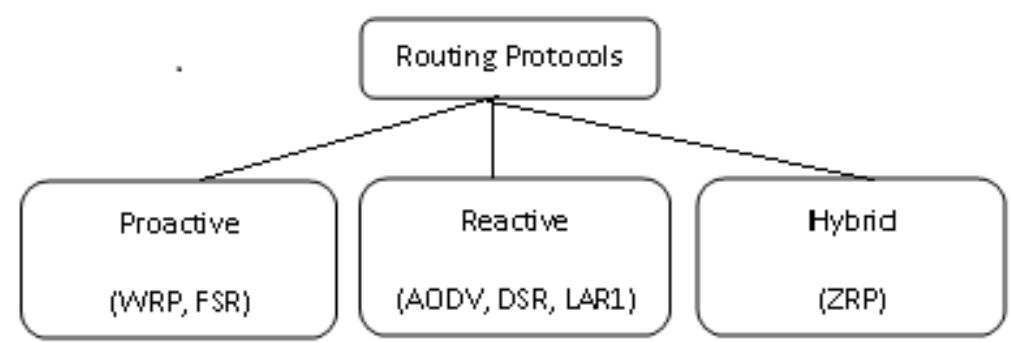

Figure 1 Taxonomy of Routing Protocols

Reactive (On Demand) protocols discovered routes only when they are needed. The main advantage of this approach is lower number of control messages but having higher latency in route discovery. A proactive (Table Driven) protocol maintains routing tables for the entire network for each node. The main advantage of this approach is its low latency in discovering new routes but it generates high volume of control messages. Hybrid protocols exploit best features of both proactive and reactive routing protocols. The proactive part of the protocol is restricted to a small neighborhood of a node and the reactive part is used for routing across the network.

\section{AD-Hoc Routing Protocols}

In this section an overview of the protocols that are most widely and frequently used in existing work are described, illustrating the properties, key strength and weaknesses.

\subsection{Ad-Hoc on Demand Distance Vector Protocol (AODV)}

AODV [1] [2] is a reactive routing protocol which is the extension of classical DSDV routing algorithm. It is designed to improve upon the performance characteristics in the creation and maintenance of 
routes. The primary objective of AODV is to broadcast route discovery packets (RREQ) only when necessary and the packet is flooded throughout the network. If the node is the destination or has valid route it unicast route reply packet (RREP) back to the source. Routes are maintained as long as they are needed by the source. Sequence number ensures the freshness of routes and guarantees the loop free routing. AODV creates no extra traffic for communication along existing links. It decreases the control overheads and reacts reactively fast to the topological changes in the network. The downsides of AODV includes that it is having stale entries and periodic beaconing leads to unnecessary bandwidth consumption. Latency and the security are its evident weakness.

\subsection{Dynamic Source Routing (DSR)}

DSR is a source routed on demand routing protocol [3] [4].The packet contains the full route to destination and the intermediate nodes do not have to make any routing decisions. Route discovery and the route maintenance are the two major phases of this protocol. Each mobile node is required to maintain a route cache and updates entries. Route maintenance is the mechanism used to detect link failure and monitors the operation of route. There is no need to periodically flood the network for updating the route tables which is an addition to its strength. Efficient only to small scale networks and the problem of reply storm arrived here.

\subsection{Location Aided Routing (LAR1)}

LAR is a uniform on demand routing protocol [5] [6].It is base on geographical information. LAR assumes that each node knows its physical location by using GPS which is further used to limit the flooded area of route request packets. The major advantage of LAR is reduced control overhead using an efficient use of geographical position information. The disadvantage is that each node must support GPS which increased the utilization of bandwidth.

\subsection{Wireless Routing Protocol (WRP)}

WRP is a proactive uniform protocol [7] and guarantees loop-free routing similar to DSDV, but differ in terms of table maintenance and update mechanisms. In WRP each mobile host maintains four routing tables i.e., distance table, route table, link cost table and a message retransmission list. $x$ Significant amount of memory and greater processing power is required. To improve reliability, every neighbor is required to send acknowledgements with respect to update packet received. Control overheads involved in WRP which makes it unsuitable for highly dynamic and large networks.

\subsection{Fish eye (FSR)}

FSR is a proactive, non-uniform routing protocol [8] based on link state routing. The goal is to provide an accurate routing solution while the control overhead is kept low by taking an advantage of global network information. It works by not sending all available link information but focusing on the network topology in the direct neighborhood of the node. It limits scalability and does not provide any security.

\subsection{Zone Routing Protocol (ZRP)}

ZRP is a hybrid protocol [9]. It is based on the idea of zones. A routing zone is defined for each node separately and the zones of neighboring nodes can overlap. The behavior of ZRP is adaptive and optimized to reduce routing overheads. It is targeted for large networks and ensures loop free routing but stale information is propagated whenever there is a high mobility.

\section{LITERATURE REVIEW}

The proposed research has been motivated by several earlier researches in the literature related to the performance comparison of ad-hoc routing protocols under varying network scenarios. In [10] using GloMoSim Simulator .AODV, WRP, DV, and DSR are compared using performance metrics end-to-end delay, packet delivery ratio, and message overhead, with the control parameters. DSR was found to have the lowest control message overhead but highest end-to-end delay of all. WRP consistently demonstrated the lowest end-to-end delay of all the protocols and had wonderful packet delivery ratio. In [11] only DSR and AODV are considered and the parameters investigated was packet delivery ratio, routing load and average end-to-end delay. DSR always showed a lower routing load than AODV, but when the MAC 802.11 was used both protocols was found to be very similar. Performance of AODV is better in terms of packet delivery ratio and delay under heavy network. In [4] AODV, DSR, and STAR are compared in terms of data delivery, control overhead, and average latency under various scenarios of mobility, connectivity density, number of data flows, domain shape and initial node placement using GloMoSim simulator. AODV turned out to be the best protocol in terms of data delivery in densely connected scenarios, whereas STAR was found to be the best performer in all the remaining cases. In [12] Cano and Manzoni studied the routing energy consumption of the Protocols AODV, DSR, TORA and DSDV under different scenarios using the ns-2 simulator. The results showed that DSR outperforms AODV 
and DSDV due to its aggressive approach in listening and caching. TORA had very high consumption due high volume of messages for route discovery and maintenance. In [13] performance comparison of AODV,DSR, ZRP and OLSR is done in realistic scenarios and out of which AODV protocol performed best with a small berm in overall throughput. In [3] AODV, TORA and DSR protocols are compared by varying data rates using the Opnet simulator with network of 20 fixed nodes only. TORA turns out to be the premium in terms of PDR, with AODV being next best with better delay and load. DSR performs worst.

\section{SimUlation ENVIRONMENT}

The overall goal of the experiments performed was to analyze and compare the performance of the routing protocols in Mobile Ad-hoc environment. The simulation has been performed in this study using the Global Mobile Information System Simulator (GloMoSim) which provides a scalable simulation environment for large wired and wireless communication networks [14][15]. GloMoSim uses a parallel discrete-event simulation capability provided by Parsec. Extensibility and the scalability are the key attributes in the design of GloMoSim. For our simulation, we constructed a 1500 x $1500 \mathrm{~m}$ square topology. The number of mobile nodes was varied from 10 to 50 . The node aggregation technique is used to give significant benefits to the simulation performance. Traffic sources are Constant Bit Rate (CBR). Only 512 byte data packets are used and the total number of packets was 20000. Simulating the networks with up to thousand nodes linked by a heterogeneous wireless communications capability and simulation is run with the light speeds using the mobility parameter which is set between 0 to $10 \mathrm{~m} / \mathrm{sec}$ so that there can be movement between the nodes. 802.11, CSMA, and TSMA was used as MAC protocol. In this section, we present the results of simulating Bellman-Ford, AODV, FSR, DSR, LAR1, WRP and ZRP by varying number of nodes.

Table 1 Simulation Parameters

\begin{tabular}{|c|c|}
\hline TERRAIN DIMENSION & $1500 \mathrm{~m} \times 1500 \mathrm{~m}$ \\
\hline NETWORK (Routing) & Bellman-Ford, AODV, Fisheye(FSR), DSR, LAR1, WRP, \\
ZRP
\end{tabular}

Different routing protocols are evaluated by using three performance metrics packet delivery ratio, end- to-end delay and throughput. Packet Delivery Ratio is the ratio of the number of packets received at destination to the number of packets sent by the source. Greater the value of PDR means better the performance of protocol. It directly or indirectly depends on route discovery time, throughput and number of hops. Average End-to-End delay is the average time taken by the packet from source to destination in seconds and lower value of it is preferred. Throughput is the total number of packet transmitted through the network in a unit of time. It is the useful data rate measure in bits per second (bps).

\section{SIMULATION RESUlTS AND DiSCUSSION}

In Mobile Ad hoc Network (MANET), mobility, traffic and node density are main network conditions that significantly affect the performance of the network. In this section simulation results for the selected protocols with respect to packet delivery ratio, average end-to-end delay, and throughput are elaborated.

\subsection{Simulation Result for Packet Delivery Ratio}

Figure 2 shows the PDR of the routing protocols for 802.11 data link and results indicates that AODV and LAR1 has maximum PDR and outperforming the rest of the protocols because in their case maximum packets are received at destination node where as Bellman-Ford has the minimum PDR. Figure 3 shows the PDR of the protocols for CSMA and it showed a repeated very up and down performance pattern and it must be noticed that LAR1 suffered a drop in performance having minimum PDR. Figure 4 shows the PDR graph for TSMA and here WRP did not see any significant delivery ratio and for the entire MAC protocols studied ZRP is having constant PDR.

\subsection{Simulation Result for Average End-to-End Delay}

Figure 5 shows the average End-to-End delay graph for TSMA and in terms of it WRP and FSR demonstrate lower delay than other protocols due to their operation which is table driven in nature. The presence of routing information in advance leads to lower average end-to-end delay. AODV shows the average 
performance. LAR1 broadcast messages through entire network to find its destination because of its reactive nature and needs more time in route discovery and hence performs worst.

\subsection{Simulation Results for Throughput}

Figure 6 throughput results for 802.11 shows LAR1 and AODV has highest throughput value than other protocols as they deliver packets at higher rates. ZRP was also superior to rest of the protocols because of proactive and reactive characteristics. In ZRP, while sending in INTRA zone routing protocol if it fails to send data or link breakdown occurs then INTER zone routing protocol will be activated. Henceforth data transfer will continue. Figure 7 and Figure 8 shows throughput for CSMA and TSMA, here also ZRP shows a significant performance due to its adaptive behavior and the ability to identify multiple routes to a destination with less number of hops and minimized route discovery time, which provides. There was a repeated mild up and down performance pattern by other protocols.

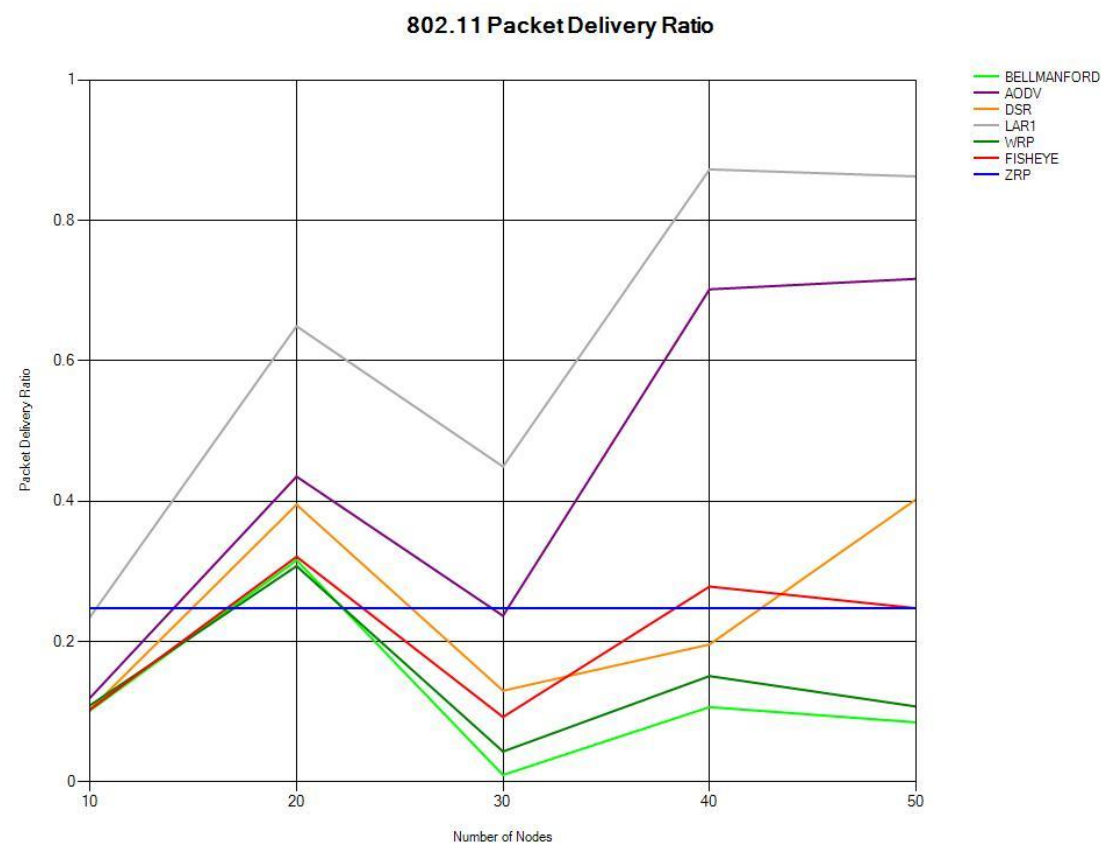

Figure 2 802.11 Packet Delivery Ratio

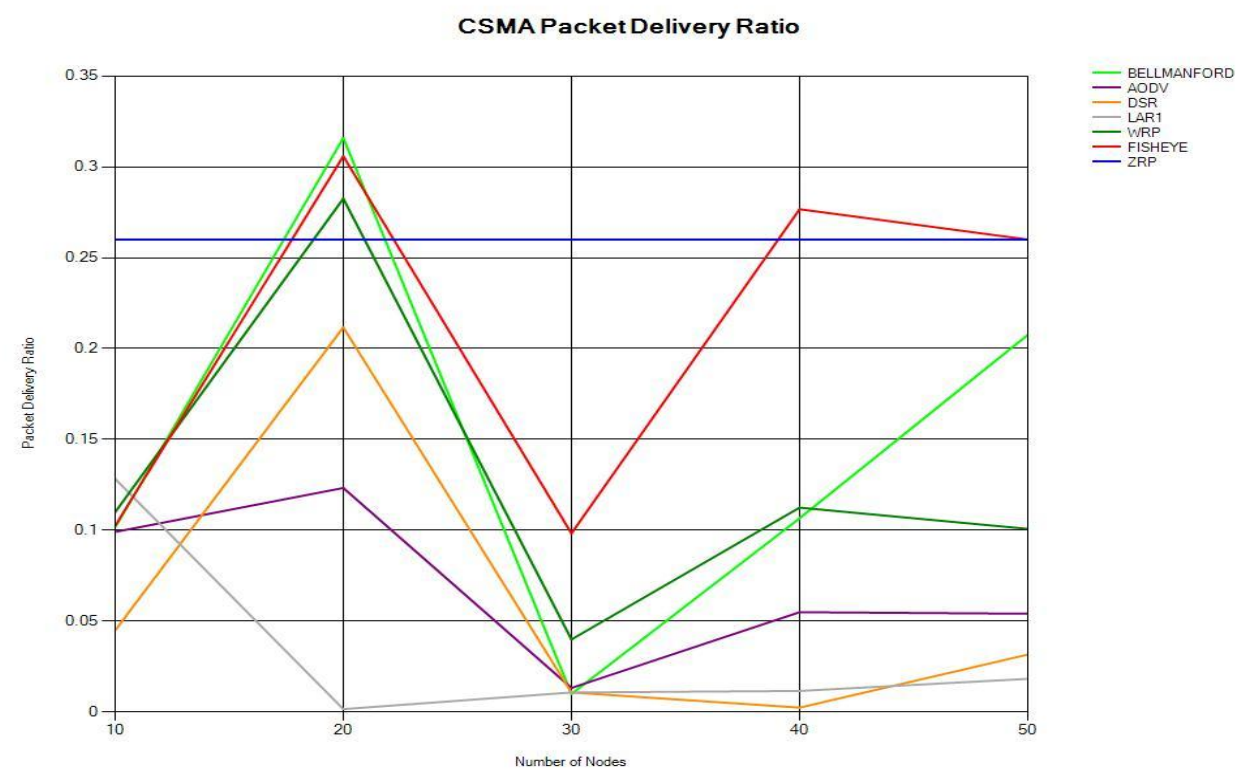

Figure 3 CSMA Packet Delivery Ratio 

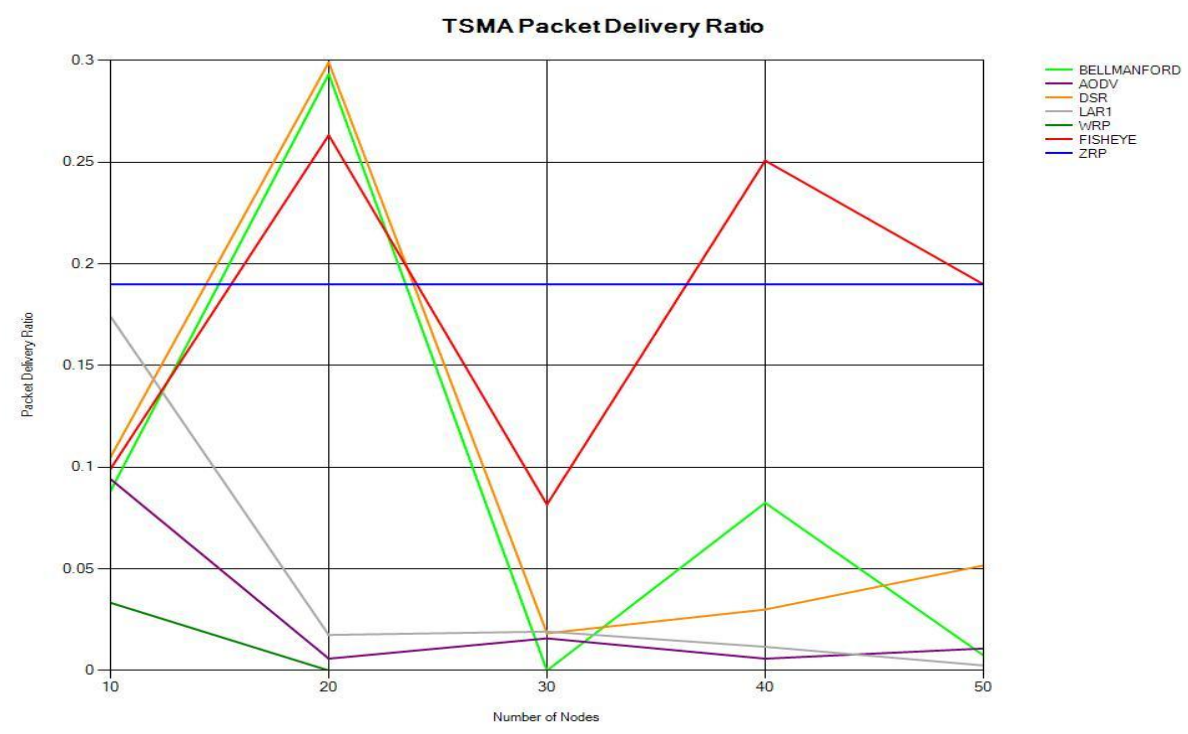

Figure 4 TSMA Packet Delivery Ratio

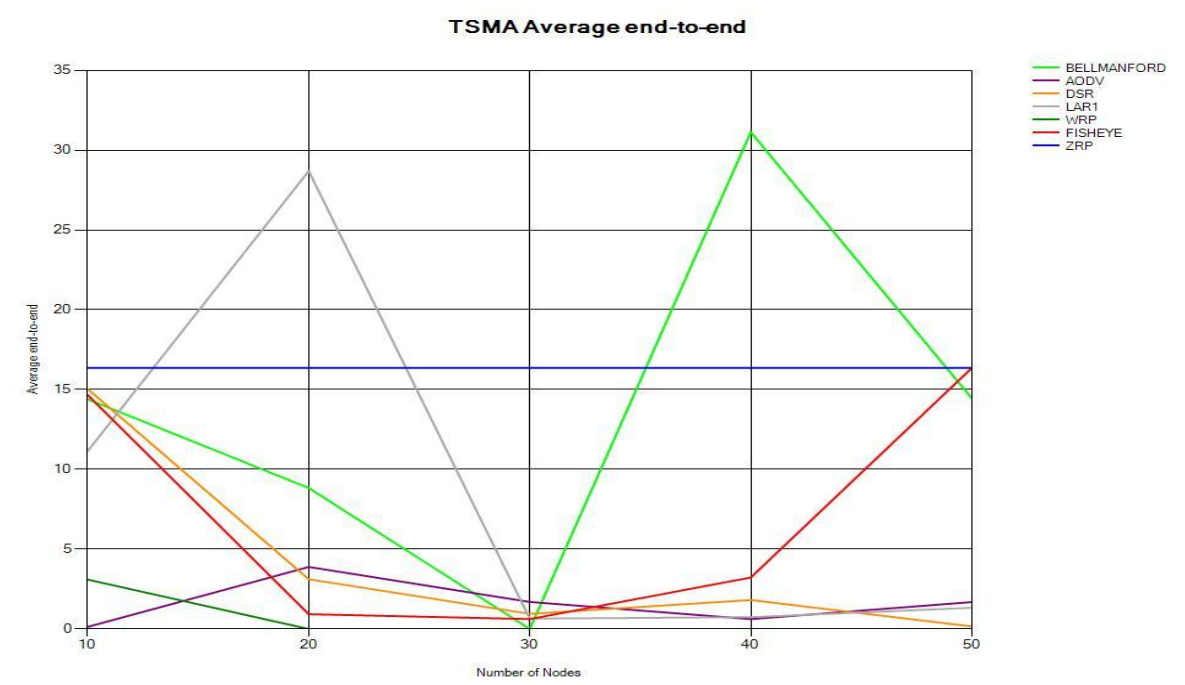

Figure 5 TSMA Average End-to-End Delay

802.11 Throughput(Server)
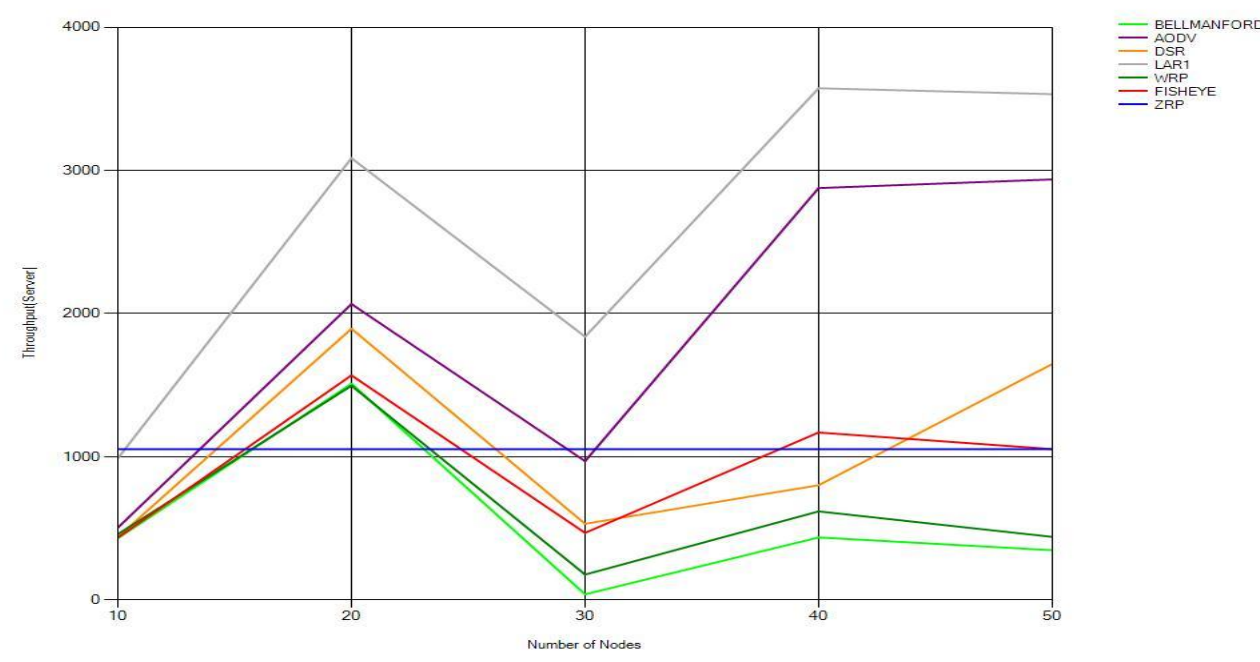

Figure 6802.11 Throughput 


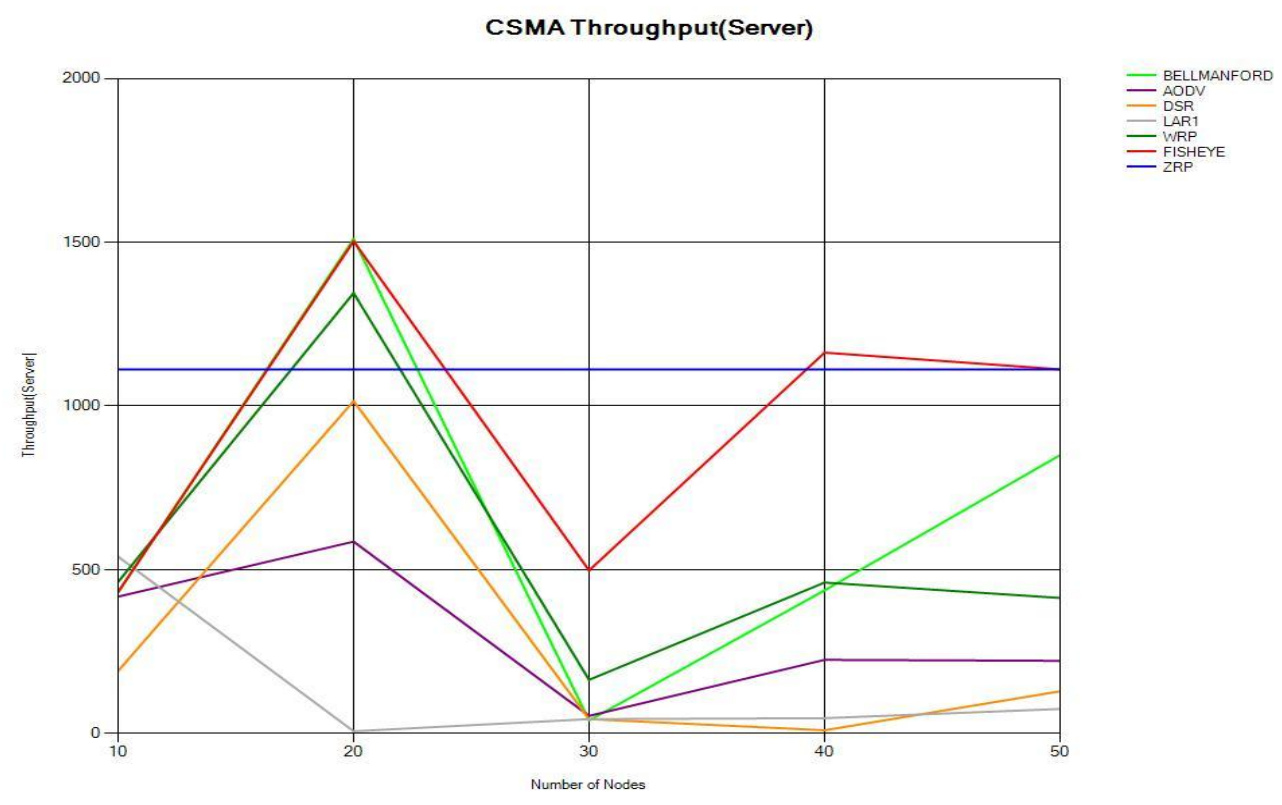

Figure 7 CSMA Throughput

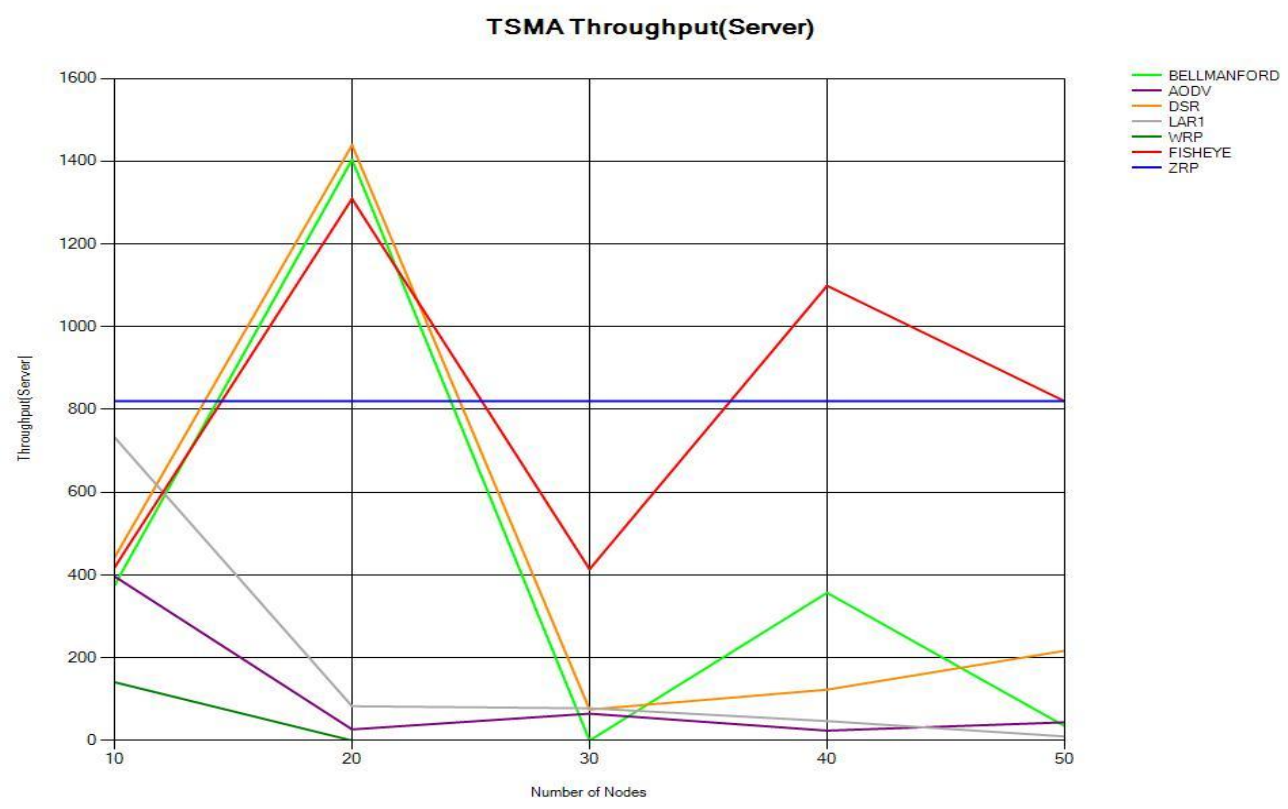

Figure 8 TSMA Throughput

\section{Conclusion}

Performance evaluation of mobile ad-hoc routing protocols is one of the valuable aspects. In this paper, Simulation demonstrates the best performance of the different protocols and revealed that combination of it can be used for good result. ZRP shows the best performance in terms of throughput compared to other protocols but for 802.11 AODV and LAR1 outperforms because they react reactively fast to the topological changes in network. WRP and FSR performed the best in terms of the average end-to-end delay as compared to rest of the protocols. FSR uses up to date shortest routes and both having the loop free routing. AODV and LAR1 displayed similar performance by its members, which was very superior to that of the other protocols in terms of packet delivery ratio for 802.11. For CSMA LAR1 suffered a drop in performance but AODV surpassed it for good. ZRP is having constant PDR throughout all the MAC protocols and leads to average performance. LAR1 and AODV had similar performance. Future directions include: evaluating the protocols with control parameters for larger network layout. We also observed that the interplay between the routing and MAC layers could affect 
performance significantly. This observation also emphasizes the critical need for studying interactions between protocol layers.

\section{REFERENCES}

[1] Perkins, C.E. and E.M. Royer. Ad hoc on-demand distance vector routing (AODV), In Proceedings of the 2nd IEEE Workshop on Mobile Computing Systems and Applications, New Orleans, LA; 1999.

[2] Perkins, C.E., E.M. Royer, and S.R. Das, Ad hoc on demand distance vector (AODV) routing, IETF Internet Draft, 2003.

[3] Johnson, D.B., D.A. Maltz, and J. Broch, DSR the dynamic source routing protocol for multihop wireless ad hoc networks, in C.E. Perkins (Ed.). Ad Hoc Networking. Reading, MA: Addison-Wesley; 2001.

[4] J.Broch,D. Johnson, and D. Maltz, The dynamic source routing protocol for mobile ad hoc networks, IETF Internet draft 2003. Available from: http://www.cs.cmu.edu/dmaltz/internetdrafts/draft-ietf-manet-dsr-09.txt.

[5] Ko, Y.B. and N.H. Vaidya., Location-Aided Routing (LAR) mobile ad hoc networks, In Proceedings of ACM/IEEE MOBICOM '98. Dallas, TX; 1998.

[6] Ko, Y.B. and N.H. Vaidya, Location Aided Routing (LAR) in mobile ad hoc networks. Wireless Networks 2000;6 (4):307 321.

[7] Lee, S.J., et al., Selecting a routing strategy for your ad hoc network in Symposium on Applied Computing, Seoul, Korea: Elsevier; 2002.

[8] Pei, G., M. Gerla, and T.-W. Chen., Fisheye state routing: a routing scheme for ad hoc wireless networks, Proceedings of the IEEE International Conference on Communications. New Orleans, LA; 2000, pp. 70-74

[9] Nicklas Beijar, Zone Routing Protocol (ZRP), Networking Laboratory ,Helsinki University of Technology ".

[10] A. K. Pandey, H. Fujinoki, "Study of MANET routing protocols by Glomosim simulator" , International Journal of Network Management, Volume 15, 2005, pp. 393-410

[11] S. R. Das, C. E. Perkins, E. M. Royer, "Performance Comparison of Two On demand Routing Protocols for Ad Hoc Networks", Proceedings of the IEEE Infocom 2000, pp. 3-12, March 2000

[12] Juan-Carlos Cano and Pietro Manzoni, A Performance Comparison of Energy Consumption for Mobile Ad-Hoc Network Routing Protocols, Proceedings, 8th International Symposium on Modeling, Analysis and Simulation of Computer and Telecommunication Systems, 2000

[13] Julian Hsu, Sameer BhatiaMineo Takai, Performance comparison of AODV,DSR, OLSR, OLSR v2 and ZRP in REALISTIC SCENARIOS".

[14] Jorge Nuevo, A Comprehensible GloMoSim Tutorial

[15] GloMoSim: Global Mobile Information Systems http://pcl.cs.ucla.edu/projects/glomosim/ 